\title{
Von der einen Nation zur kulturell vielfältigen Region. Der „spatial turn“ als Provokation der Nationalphilo- logien
} Co przynosi germanistyce „spacial turn“, wzmożone zainteresowanie kategorią prze-
strzeni, tak obecnie nośną w międzynarodowym dyskursie naukowym? Najpierw po-
dejmiemy próbę konkretyzacji tej kategorii w odniesieniu do historii niemieckiej (a
częściowo także polskiej), skupiając się przy tym na pojęciach ,przestrzeń, ,naród“,
,region“ oraz ,semiosfera'. Po jakimś czasie okaże się, że „spacial turn“ jest niezwykle
produktywnym wyzwaniem dla filologii (także dla germanistyki), których przedmiot(y)
badań definiowane są narodowo. „Spacial turn“ narusza podstawowe aksjomaty filo-
logii, prowadzi do ich rozluźnienia.

Was bringt der „spatial turn“, die im internationalen Wissenschaftsdiskurs z.Z. akute „,äumliche Wende“ der Germanistik? Er wird hier zunächst auf die deutsche (und z.T. auch polnische) Geschichte hin konkretisiert. Im Mittelpunkt werden dabei Begriffe wie ,Raum', ,Nation', ,Region' und ,Semiosphäre' stehen. Nach einigen Umwegen wird sich dann zeigen, dass der „spatial turn“ eine produktive Herausforderung für die auf national definierte Gegenstandsbereiche ausgerichteten Philologien (wie eben auch die Germanistik) darstellt. Er kratzt an ihren Grundaxiomen, lockert sie auf.

What will the ,spatial turn“ in international scholarship discourse add to the Germanic Studies? The term will first be specified in regard to German (and partly also Polish) history. The focus will be on such concepts as ,space', ,nation', ,region' and ,semiosphere'. After a few detours, it will then become clear that the „spatial turn“" provides a productive challenge for the philology of nationally defined areas of research such as Germanic Studies. It teases with its basic principles and loosens them up.

Paradigmenwechsel (KUHN 1973) in Kultur und Wissenschaften beginnen oft gleichzeitig an mehreren verschiedenen Orten unabhängig voneinander. Inkonsistenzen in den vorherrschenden Theoriegebäuden, Unzeitgemäßheit der me- 
thodischen Ansätze, Enge und bereits abgestandene Denke in bisher dominanten Beschreibungsmodellen provozieren von sich aus vielerorten ähnliche Diskussionen, die sich, aus verschiedensten Quellen gespeist, langsam zu einer erkennbaren Bewegung massenhaften Zweifels und Einspruchs vereinen. Aus dem Rückblick erscheint es dann manchmal als erstaunlich, wieviel unabgesprochene Übereinstimmung es an oft über die ganze Welt verstreuten Orten gegeben hat - erklärbar wohl nur aus ähnlichen Rahmenbedingungen und gemeinsamen Herausforderungen, die zu analogen Reaktionen und Bewältigungsstrategien führen mussten. Im Nachhinein erhalten solche Strömungen dann Bezeichnungen, die sie als Einheit erscheinen und die Vielfältigkeit ihrer Ursprünge leicht vergessen lassen.

So ist es auch mit dem ,spatial turn“ (BACHMANN-MEDICK 2006), der ,Wiederkehr des Raumes“ (SCHLÖGEL 2005, vgl. aber auch schon PIEPER 1987), von dem in den letzten Jahren unter oft unterschiedlichen Bezeichnungen so oft zu hören und zu lesen war (z. B. WEIGEL 2002). Als aus vielerlei Richtungen gespeiste, in sich durchaus auch widersprüchliche Bewegung, lässt er sich im Kern auf zwei Grundtendenzen zurückführen, die, gerade weil sie kontradiktorisch zueinander stehen und einander fast schon gegenseitig ausschließen, die Denkpole eines dialektischen Wechselspiels darstellen, das den „spatial turn“ überhaupt erst fruchtbar macht. Nicht diese beiden Ansätze machen ihn aus, sondern die durch die Spannung zwischen ihnen provozierte geistige Tätigkeit. Da heißt es einerseits (SCHLÖGEL 2003:9-78), im internationalen wissenschaftlichen, kulturellen und gesellschaftlichen Diskurs habe es lange Zeit eine Vernachlässigung der Kategorie ,Raum‘ gegeben, weil die allgemeine verkehrs- und kommunikationstechnische Beschleunigung (BAIER 1990, 2000; GÖTZE 2004:288-297) den ,Raum“ (hier verstanden als bloße räumliche Ausdehnung) nur als immer schneller überwindbaren Störfaktor habe erscheinen lassen, den es möglichst rasch zu durchqueren gelte. ,Raum' sei dadurch ein bloß Negatives geworden. Dies habe sich nun geändert durch die unvorhersehbare Koinzidenz so unterschiedlicher Entwicklungen wie der abermals entgrenzenden „Globalisierung“ ökonomischer, politischer und kultureller Praktiken in der „Einen Welt“ nach dem Ende des Kalten Krieges, die als Gegenbewegung die von ,unten“, der ,Basis“, den ,grassroots“ ausgehende Stärkung zuvor schon totgesagter Regionen und partikularer Kulturen provoziert habe (POTT 2002), aber auch durch die Entwicklung von Internet und Cyberspace (FUNKEN / LÖW 2003), die als abermalige Kommunikationsbeschleuniger das scheinbare „Verschwinden des Raumes“ (FRIEDMAN 2008:20-22) mit weitreichenden ökonomischen und sozialen Folgen so weit vorantrieben, dass die damit entstandenen machthaltigen Kommunikationsnetzwerke ebenso wie die 
weiten Räume, die sie aus sich ausschlössen, eine nicht zuletzt auch räumlich orientierte Analyse herauszufordern begännen (CASTELLS 1996:98). Der „spatial turn" verweise auf die bedrohte Widerständigkeit kleiner Lebenswelten unterhalb global alles vereinheitlichender Vorgänge und Zusammenhänge und fordere zu einer erneuten Reflexion des Raumes heraus.

Seine polnische Variante wäre nach diesem Verständnis des „spatial turn“ die „Entdeckung“ der Regionen durch Schriftsteller wie KAZIMIERZ BRAKONIECKI (1998), Stefan ChWin (1995), PaWet Huelle (1987), ARTUR Daniel LisKowACKI (2000), ANDRZEJ STASIUK (1995), Olga TOKARCZUK (1999) oder ADAM ZAGAJEWSKI (1998) oder den Verein „Borussia“ (OREOWSKI 2002), die in der polnischen Germanistik (und in den polnischen Geschichts-, Kultur- und Geisteswissenschaften überhaupt) begleitet wird von einer Besinnung auf die multikulturelle Geschichte vieler erst seit 1945 polnischer Regionen, während ihm in Deutschland die in Film, Literatur und Alltagskultur vor allem in den 80er und 90er Jahren auffällige Welle neuer ,kritischer ", Heimat"Darstellung (man denke an Edgar Reitz' Filmfolge „Heimat“, an Heimat- und Erinnerungsliteratur aus Sicht der ,kleinen " Leute von ,unten " oder die bis in Volkshochschulen und lokalhistorisch orientierte Freizeitclubs hineinreichende oral-history-Bewegung) entspräche (STRZELCZYK 1999). Gegenstand des ,spatial turn“" wäre demnach eine Räumlichkeit, die sich dann (aber nur unter diesem Aspekt!) eins zu eins in den Begriff ,Region(alität) ' in dem Sinne übersetzen ließe, dass diese eine lokal konkrete Außen- und Gegenwirklichkeit gegenüber globalen Trends, „Textualismus und Kulturalismus“ (BACHMANN-MEDICK 2006:297) impliziere, also „dass es Örter gibt: Örter, also nicht bloß Symbole, Zeichen, Repräsentationen von etwas [...] Städte, die getroffen werden können, Türme, die zum Einsturz gebracht werden können“ (SCHLÖGEL 2004:262).

Doch der „spatial turn“ betreibt nicht einfach antiglobalistischen Gegen-Regionalismus im Stile von KoHR (1983), der in eine substantialistische „Raumfalle" (LIPPUNER / LOSSAU 2004) und geopolitisch inspirierte Phantasien von einem Clash of Civilizations (HUNTINGTON 1996) führen kann; vielmehr wird unter seinem Vorzeichen und entgegen dieser eher substantialistischen Verwendung von ,Raum' zugleich auch versucht, die Kategorie, Raum' unter den Bedingungen einer Postmoderne und einer globalisierten Welt neu zu denken, die viele ,Raum'-Konzeptionen und naiv wahrnehmungsrealistische Annahmen über den ,Raum' als illusionäre Konstruktionen erscheinen lassen. So hat etwa die Erforschung der „mental maps“ (vgl. etwa GouLD / WHITE 1986 oder DAMIR-GEILSDORF / HARTMANN / HENDRICH 2005), der Art und Weise, wie Menschen sich die Welt vorstellen, dazu geführt, dass ,Raum' als eine auch 
subjektiv rezipierte Größe mit massiven Abweichungen von der objektiven, realen Geographie erfassbar wird. Beispielsweise glaub(t)en viele Österreicher im Zuge der einstigen Aufteilung Europas in zwei nach Himmelsrichtungen benannte politische Blöcke, Prag liege östlich von Wien ([ANONYM] 2006:1).

,Raum“ ist spätestens seit der Einführung der modernen Verkehrsmittel nicht mehr jene homogen in sich geschlossene Größe, als der er selbstverständliche Grundlage schutzbedürftigen Sich-Beheimatet-Fühlen-Wollens lange Zeit war (BACHELARD 2003), sondern als Lebenswelt vielfach verzerrt, zersplittert und zerrissen (Löw 2001). Der homogene ,Raum', den die auf gleichmäßige Proportion und räumliche Harmonie ausgerichtete Renaissancemalerei als optischen Normalfall zu etablieren gewusst hatte, erweist sich als artifizieller, mühsam konstruierter Ausnahmefall, die von verzogenen, vielfach sich überschneidenden Perspektiven scheinbar verwirrte Malerei des Mittelalters (oder dann auch wieder des Expressionismus) als dem menschlichen Erleben des ,Raums' viel angemessener. Mit solchen Einsichten restituiert diese Richtung des „spatial turns“ den ,Raum'-Begriff nicht, sie dekomponiert ihn. ,Raum“ zerfällt in Teile, die zuvor nicht als solche erkennbar waren (wie etwa mentale Karte und objektive Geographie) und erscheint nicht mehr als stabile Größe, die menschliches Beheimatet-Sein in einem unverrückbar bergenden, behälterähnlichen Außerhalb aller Kultur birgt, „Raum meint soziale Produktion von Raum als einem vielschichtigen und oft widersprüchlichen gesellschaftlichen Prozess, eine spezifische Verortung kultureller Praktiken, eine Dynamik sozialer Beziehungen, die auf die Veränderbarkeit von Raum hindeuten." (BACHMANNMEDICK 2006:289) „Raum“ ist soziale Praxis, „Raumkultur“ (LÄPPLE 1991; DANGSCHAT 1996; STURM 2000; LÖW 2001; ROLSHOVEN 2003), quasi dreidimensional gewordene Macht und Hierarchie, Weltdeutung und Interaktionsgefüge (MARESCH / WERBER 2002). Räume (in diesem Sinne) sind nicht, sie werden gemacht (SCHULTZ 1997, 2002; SCHENK 2004).

Selbst der scheinbar so naive Begriff ,Heimat', mit dem ,Region' gerne fälschlich gleichgesetzt wird (als gäbe es nicht auch unheim[at]liche Regionen, Arbeits- und Vernichtungslager, Elendsviertel, Fabrikhallen, Abraumhalden oder Orte der Abwesenheit und des sinnleeren Wartens wie Hotelhallen und Einflugschneisen), erweist sich unter dieser Perspektive als eine topischen Regeln folgende soziale und poetische Konstruktion einer bestimmten Art von Raum, eines Traum-, eines Nicht- und Gegen-Raums, einer U-Topie gegen als bedrohlich empfundene Wirklichkeit - eine konservative Variante des „Thirdspace“ (SOJA 1996), des „,dritten Ortes“, der von Wirklichkeit durchsetzten gegenwirklichen „Heterotopie“ (FoUCAULT 2005), mit der im Zuge des „spatial 
turn“ Orte umschrieben werden, die mit einer zusätzlichen Bedeutungsdimension versehen wurden, die aus der bloßen Wirklichkeit hinausreicht, so dass ihre Bewohner oder Besucher sich nicht nur in der realen, sondern zugleich in einer „,imaginären Geographie“ (PEKAR 1998) bewegen.

Im globalen Kontext am wirkungsvollsten und spektakulärsten war die Wirkung des ,spatial turn“ in den ,(post-)colonial studies“ (ASHCROFT / GRIFFITHS / TIFFIN 1989), in denen nach einer Welle intensiver Kolonialismuskritik (insbesondere SAID 1994) nach dem dialektischen Wechselverhältnis zwischen Kolonialisierten und Kolonisierern gefragt wird und danach, wie der Kolonialismus beide verändert hat (BHABHA 2000). Ausgehend vom Verhältnis zwischen Zentrum und Peripherie, Europa und seinen (einstigen) Kolonien wurde dabei (erneut) eine Frage aufgeworfen, die mit teilweise anderer Terminologie das Thema „Regionalität“ auch in Mitteleuropa bereits spätestens seit dem 19. Jhd. bestimmt hatte, die Frage nach dem Verhältnis zwischen sich als universal inszenierenden Instanzen in den Zentren der Macht, der Planung und des dominanten Denkens einerseits und der von dort aus als partikular marginalisierten Lebenswirklichkeit der vielen nicht mit den Konzepten und Planvorgaben des Zentrums einverstandenen oder kompatiblen Menschen andererseits, die vom Zentrum aus als bloß zu überwindende Abweichung Zurückgebliebener in der Provinz erscheinen (wobei die Zentren sich selbst als Instanzen der Modernisierung betrachten).

Diese Spannung zwischen Peripherie und Zentrum findet im deutsch-polnischen Kontext ihre reale Entsprechung im kolonialen Germanisierungsdiskurs (KETELSEN 2006) der wilhelminischen Ära, in dem von Seiten der Germanisierer aus versucht wurde, alles „Nicht-Deutsche“, insbesondere die polnische (aber auch die sorbische und litauische) Sprache und Kultur der Minderheiten innerhalb des Deutschen Reiches als ein Rudiment aus ,vormodernen ' Zeiten darzustellen, das die ,noch nicht“ assimilierten Menschen, die an ihrer ,abweichenden" Identität festhielten, nur unnötig belaste und am wirtschaftlichen und sozialen Aufstieg hindere (ORŁOWSKI 1996). Dieses Argumentationsmuster gab es interessanterweise im - keineswegs konfliktfreien - Umgang mit der französischen Minderheit im „Reichsland Elsass-Lothringen“ nicht; man mochte die deutsche „Kultur“ der französischen „Zivilisation“ entgegenstellen (MANN 1918), deutsche „Kultur“ vielleicht sogar für besser halten als französische „Zivilisation“, niemand aber wäre auf die Idee gekommen, den Franzosen den Status eines „Kulturvolks“ abzusprechen und von ihnen zu erwarten, dass sie sich durch die Bereitschaft, sich kolonialisieren bzw. germanisieren zu lassen, zu einem solchen hinauf-kultivieren lassen sollten, wie es von 
den Polen, Sorben und Litauern im Deutschen Reich verlangt wurde. Für die Franzosen gab es in den Schulen denn auch muttersprachlichen (französischen) Unterricht, während den Polen, Sorben und Litauern ein solcher verweigert wurde. Sie sollten nicht nur Bewohner des Territoriums des deutschen Staates, sondern auch Mitglieder der in ihm herrschenden Kultur und Gesellschaftsordnung sein, während die Franzosen zähneknirschend als dem „Erbfeind“ zugehörig anerkannt und respektiert wurden. Dieses Beispiel zeigt, dass es in den vom „spatial turn“ betrachteten Problemen keineswegs nur um ,Räume“ oder ,Regionen“ in einem geographischen Sinne geht, sondern auch und vor allem um sozial konstruierte und d.h. lebensweltlich wirksame ,Räume', sprich: um die Strukturen, die das Zusammenleben von Menschen an Orten und in Gebieten mitgestalten und formen, um Anpassung und Widerspruch, Eigenheit und Konformität.

Für die europäische, die deutsche und polnische Geschichte ist in dieser Hinsicht die Entstehung jener Nationalstaaten von nach wie vor großem Interesse, die nicht nur das Gebiet Europas territorial unter sich aufteilten, sondern auch die Menschen so zu erziehen versuchten, dass sie sich in das jeweilige Territorium und seine Ordnung, den ,Raum` der Nation möglichst reibungsfrei einpassten. Für alle Nationen Europas war es im Zuge ihrer Konstitution seit dem 18. bzw. 19. Jhd. selbstverständlich, sich als eine in sich geschlossene, möglichst homogene Einheit zu begreifen, die einen quasi „natürlichen“ Anspruch auf ein, auf ihr Territorium erhob, in dem es dann logischerweise keine Anderen geben durfte, die das nationale Einheitspostulat durch ihre bloße Existenz in Frage zu stellen geeignet gewesen wären. Die Widersprüchlichkeit des Ganzen zeigt sich schon darin, dass es in Deutschland nicht selten dieselben Personengruppen waren, die von Polen, Litauern, Sorben, aber auch Dänen und teilweise sogar Franzosen Assimilation forderten und gleichzeitig die großenteils bereits assimilierten Mitbürger jüdischer Herkunft mit nun rassistischen Argumenten aus der imaginierten Gemeinschaft (ANDERSON 1983) der Nation wieder auszugrenzen versuchten. Dieser Umgang von Systemen mit ihrer Bevölkerung bedarf hier einer genaueren Betrachtung, die auf den ersten Blick von ,Raum'-Problematik und ,Regionalität' hinwegzuführen scheint, tatsächlich aber nötig ist, wichtige terminologische Entscheidungen und Auswirkungen des ,spatial turn“ zu verstehen.

Das Andere muss aus Sicht nationaler Ordnungsinstanzen ans Eigene assimiliert und dieses wiederum ständig daraufhin überprüft werden, ob es auch aus nichts besteht, was sich einem Anderen zuordnen ließe. Als typisches doublebind-Verfahren in einer real aus vielfältig bunter Herkunft zusammengewür- 
felten Bevölkerung von auch ansonsten äußerst heterogener Beschaffenheit erhöht dies die Anpassungsbereitschaft - fast jeder könnte ja unversehens einem Anderen zugerechnet werden, zumal die Summe der Menschen, die etwa in Wilhelminien von der Reichsregierung im Laufe der Jahre als „,vaterlandslos" bezeichnet worden waren (Katholiken, Linksliberale, Sozialdemokraten, Juden, Polen) den mit Abstand größeren Teil der Bevölkerung ausmachte. Kaum jemand war davor sicher, plötzlich als Anderer diffamiert zu werden. Vereinheitlichung ist das Geschäft des modernen Massenstaates, drohender Ausschluss ein wichtiges Disziplinierungsmittel: Niemand kämpft härter für das angeblich Eigene der Nation und leidenschaftlicher gegen das Andere als der, der befürchten muss, selbst diesem Anderen zugerechnet zu werden und sich deshalb ständig unter Beweis stellen muss, ja sich sogar dazu verpflichtet fühlt, das Andere in sich (und seiner nächsten Umgebung, oft sogar seiner eigenen Familie) zu bekämpfen. Nationalität erweist sich als wirkungsvolles Vereinheitlichungsmedium, weil sie zugleich Assimilation und ,Säuberung', Anpassungsdruck und ständige Kontrolle der Angepassten zulässt.

Diesem Druck zu entkommen gibt es nur zwei Strategien: möglichst vorbehaltlose Identifikation mit der Identifikationsvorgabe (die freilich vom diffusen Bewusstsein nicht befreien kann, möglicherweise nur illegitim dazuzugehören, und die gerade deshalb den Fanatismus derer unterstützt, die die ihnen selbst zweifelhafte Legitimät ihrer Identitätskonstruktion immer aufs Neue beweisen zu müssen glauben) oder oppositionelle Gegen-Idenität, also gegenidentitäres, vielleicht sogar gegen-identifikatorisches ${ }^{1}$ Bekenntnis zum AndersSein (wie es auch die regionalistische Parteiung des „spatial turn“ auszeichnet), das so gerade durch die Vereinheitlichungsbestrebungen der Nationen provoziert wird (und unvermeidlich zu ihnen gehört). Die noch in der 2. Hälfte des 20. Jhd.s akuten Konflikte um das Baskenland, Korsika oder Nordirland zeigen, wie selbst in solchen Staaten, die mit bereits abgerundetem, eigenen ${ }^{\text {‘ }}$ Staatsgebiet und scheinbar, sicherer' nationaler Beschaffenheit in das Zeitalter des Nationalismus eingetreten waren, die Umdeutung des Herrschaftsgebietes eines Fürstenhauses in das staatliche Gehäuse einer Nation zu Spannungen mit Bevölkerungsgruppen führen musste, die sich der Nation nicht zurechneten und dieser gegenüber auf einer eigenen Identität (und einem eigenen Territo-

Gegen-identitäre Identitätsbildungen locken mit Anschluss an Gegen-Kollektive, innerhalb derer sich freilich dasselbe Problem stellen kann, während gegen-identifikatorische Identitätsbildung den Identitäts- und Identifikationsprozess als Ganzes in Frage stellt und sich in das reflektierte Bewusstsein brüchiger Identität zurückzieht. 
rium) beharrten. Diese Spannungen wurden aus Sicht der ethnischen Minderheiten bereits im 19. Jhd. als analog zu der erfahren, die die Kolonialgebiete durchzogen (SAID 1994:193) - und tatsächlich waren die Versuche zur nationalen Assimilierung ethnischer Minderheiten nichts anderes als Maßnahmen der ,inneren Kolonialisierung“ und „Binnenkolonialisierung“ (SERING 1893), wie man das in Preußen nannte. Diese richtete sich nicht nur gegen ethnische, sondern auch gegen politische und regionale (teilweise sogar noch - man denke an den Kulturkampf - konfessionelle) Abweichung - in Deutschland z. B. wurde lange Zeit der Dialekt bekämpft - und war im Prinzip nichts anderes als ein Versuch der Vereinheitlichung (und Mobilisierung) der Bevölkerung, die von allen Identifikation, Teilnahme und Opfer an den Vorgaben des einen Zentrums in Berlin verlangte, Gleichheit zugleich forderte, versprach und androhte.

Die vielen historisch gewachsenen lokalen und regionalen (und auch ethnischen) Sonderidentitäten in dem von Preußen bunt zusammeneroberten Raum erforderten - wenn man denn nationale Vereinheitlichung wollte - besonders intensive Vereinheitlichungsmaßnahmen. Die Neigung gerade des deutschen Nationalismus zur Radikalität hängt mit der Brüchigkeit seiner Bevölkerungsbasis zusammen. Die Nation musste entsprechend stark behauptet und ihre Existenz dadurch bewiesen werden, dass sie denen möglichst intensiv erlebbar wurde, die zu ihr hinzugerechnet wurden. Kaum jemand konnte sich ja den ,Raum` der Nation, die vielen Menschen und das große Gebiet, aus dem er bestehen sollte, als Ganzes vorstellen und doch sollte jedem Einzelnen in ihm das proklamierte Kollektiv namens „Nation“ als , sein“ Raum erscheinen. Nationalisierung bedeutete deshalb in erster Linie Integration der Menschen in den Vorstellungsraum, die imaginäre Geographie von ,Deutschland ' und dem deutschen ,Raum‘. Bücher, die den Deutschen Deutschland vorstellten, damit sie sich ihre „Heimat“, die angeblich ihr „Eigenes“ war, zumindest im Geiste aneignen konnten, gehörten $\mathrm{zu}$ den wichtigsten Medien des Nationalstaates: Atlanten, Bilderbücher, Landeskunden, Deutschlandführer, Das ist des Deutschen Vaterland (KüRSCHNER 1896). Der Bayer konnte sich so mit der Kurischen Nehrung als einem Stück „Heimat“ anfreunden, der Ruhrpottkumpel staunend von der Kulturstadt München lesen, zu der den geforderten emotionalen Bezug aufzubauen ihm sicher ebenso leicht gefallen sein dürfte wie dem polnischsprachigen Oberschlesier, sich mit der deutschen „Kolonialarbeit“ in Deutsch-Südwestafrika zu identifizieren. Nation und Nationalstaat waren abstrakte Größen, der Raum, in den man sich hineinimaginieren sollte, trotz des neuen Verkehrsmittels Eisenbahn für kaum jemand ein wirklich angeeigneter ,eigener' Raum. Man musste seine Vorstellung lernen. 
Zu den wichtigsten politischen Konzepten der Zeit gehörte deshalb die Erfahrbar-Machung des Ganzen für den Einzelnen, damit dieser möglichst nahtlos in ihm aufgehen, von den Verwaltern des Ganzen in diesem Ganzen bewegt, eingesetzt und genutzt werden könne. Heute hat man für diese Zwecke auf europäischer Ebene den Schüleraustausch, dessen Ziel es ist, Europa, das größere Ganze, durch möglichst selbstverständlich wirkende Raumdurchquerungen möglichst vielen Heranwachsenden zu vermitteln. Damals gab es dafür das Militär, die, wie es häufig genannt wurde, ,Schule der Nation“. Eine Funktion der Armee in Deutschland war es, mit Hilfe der Wehrpflicht Menschen durch das Land zu bewegen und ihnen ein gemeinsames Erleben desselben und der erlebenden Gemeinschaft in der Nation zugeordneten Erlebnisgruppen zu ermöglichen. Männer, die ansonsten weit verstreut voneinander in verschiedenen Teilen des Reiches lebten, wurden vom Militär zu nationalen Erlebniseinheiten durcheinandergemischt und durch ihre Stationierung abseits ihrer heimatlichen Dörfer um Deutschland-Erfahrung bereichert und so zu einem Bindeglied zwischen Region und Nation (vgl. etwa die Eingangsszene von KURPIUN 1909), das zugleich, so die Hoffnung, nach seiner Rückkehr als militärisch veredelter Reservist verändernd, nationalisierend auf die Region zurückwirken sollte. Die Regionen, die vielen kleinen Lebenswelten, aus denen die Soldaten kamen, wurden dadurch zugleich eingebunden ins größere Ganze und auf dieses hin überwunden, der Einzelne aus ihnen herausgeholt in eine nationale „Bewegung“ (der er auch nach seiner Rückkehr treu bleiben sollte), die den größeren Raum der Nation als befreiend weite Bewegungsmöglichkeit gegen die Enge der von tausenderlei Rücksichten, Nöten, Pflichten und Ungleichheiten geplagten Herkunftsorte stellte. Der Kriegsausbruch von 1914 versprach mit seiner aus dem Land hinausführenden offensiven Bewegung in noch zu eroberndes Land Befreiung sogar aus den Unterscheidungen und Diskriminierungen der preußisch und protestantisch dominierten Klassengesellschaft ,hinten “ nun im ganzen Deutschland; nicht umsonst wurde trotz des katastrophalen Kriegsverlaufs der Krieg von vielen Frontsoldaten während der Weimarer Republik nachträglich zum nationalen Gemeinschaftserlebnis nostalgisiert, das angeblich alle Unterschiede nivelliert habe. Auf dieser Basis konnte dann der Nationalsozialismus mit dem Versprechen operieren, mit ihm, genauer: mit seiner „Bewegung“, seiner deshalb auch immer weiter und weiter fortzusetzenden „Aktion“ (bald wieder von der alten „Heimat“ fort in neu zu erobernde Länder) würden die Deutschen als Deutsche gleich in der nun endlich gemeinsamen einen Nation (und „Rasse“). In Deutschland hatte dies zur Folge, dass in den doch eigentlich statischen Begriff, Raum' schließlich sogar der bewegende Imperativ eingeschrieben schien, ihn zu besitzen und, war er noch nicht 
deutsch, ihn zu erobern (KÖSTER 2002). Die nationale und militärische Mobilisierung durfte die Menschen nicht zur Ruhe kommen lassen, konnte doch nur stete Bewegung in eroberten Ländern sie davor bewahren, in ihre alte Unterschiedlichkeit an Herkunftsorten zurückzusinken, die der neuen Gleichheit der „Herrschaftsmenschen“ nicht mehr entsprechen konnte. Die Alternative „Endsieg“ oder Katastrophe schloss denn auch die Möglichkeit der Rückkehr in ein vornationales Vorher prinzipiell aus (der Heimat-Kitsch der Nachkriegsjahre lebte dann von der Vortäuschung dieses Vorher bei gleichzeitigem Bewusstsein seiner unwiederbringlichen Zerstörung).

Auf polnischer Seite hatte die ihres Staates beraubte und zwischen den Teilungsmächten aufgeteilte Nation lange Zeit kein politisch ähnlich handlungsmächtiges Zentrum wie die preußische Herrschaftszentrale in Berlin. Sie konnte die Nation nicht militärisch mobilisieren und musste ihr dennoch Vorstellungen ihrer Einheit vermitteln, weshalb die ,Mobilisierung ' der polnischen Nation in erster Linie in Form kultureller Arbeit am nationalen Begehren und Imaginationsvermögen erfolgte. Der Großraum der erträumten Nation wurde nicht durch militärische, sondern ,nur' durch kulturelle Mobilisierung vorstellbar. Zwar hat es solche Kulturarbeit auch auf deutscher Seite gegeben, mit ihrer Grundlegung im regierungsamtlich verwalteten Schulunterricht erfolgte sie jedoch prinzipiell von ,oben“ und war oft noch dazu gezielt angelegt als Vorbereitung auf das Militär, das die Nation dann sinnlich als Abenteuer und Erlebnis erfahrbar machen sollte, als Einheit. Die Vertreter der polnischen Nationalbewegung hingegen mussten in den polnischsprachigen Gebieten innerhalb des Deutschen Reiches von ,unten“ aus ein polnisches Gegen-Bild entwerfen. Polen wurde nicht im Militär, sondern bestenfalls in den Sokóls, den nationalen Turn- und Sportvereinen erfahrbar, deren Feste überregionale Treffen engagierter Mitglieder der Nationalbewegung ermöglichten. Innerhalb dieser Bewegung - und später dann im polnischen Nationalstaat - konnte es kaum Platz für die Betonung regionaler Besonderheit geben; auch auf polnischer Seite wurde regionale Differenz von Seiten der Nationalbewegung aus eher marginalisiert, teilweise (wie im Fall der Kaschuben) in Frage gestellt, teilweise (wie im Fall Oberschlesiens) sogar gezielt abgeschliffen (WANATOWICZ 1991). Weil die polnische Nationalbewegung zudem ausschließlich vom freiwilligen Mittun überzeugter Anhänger lebte (während Schul- und Wehrpflicht im Deutschen Reich für alle - auch für Polen, Dänen und Litauer, Juden, Katholiken und Sozialdemokraten - galt), gab es in ihr auch wenig Platz für abweichende Differenz. Wer sich nicht in ihr aufgehoben fühlte, brauchte ja nicht mitzumachen. Deshalb ist die Betonung regionaler Eigenart innerhalb und gegenüber der Nation in Polen weit schwächer ausgeprägt als in 
Deutschland, wo der von ,oben " verordnete Zwang immer auch Opposition von , unten " und aus den Regionen provozierte.

Die polnische Entdeckung der Region als einer Quelle gegen das Zentrum gerichteter regionaler Identitätsfindung, die polnische Version des „spatial turn“ begann deshalb im Prinzip erst im Umfeld der politischen Wende von 1989/90 als Reaktion auf den kommunistischen Zentralismus, der den so lange ersehnten polnischen Nationalstaat usurpiert und die Lande mit einer offiziellen Darstellung des Lebens in ihm mit dem Ergebnis überdeckt hatte, dass die jeweils lokale und regionale Lebenswirklichkeit oft schon von sich aus Widerspruch und Nicht-Übereinstimmung bedeuten musste. Mit dem Umbruch von 1989 begann denn auch zugleich und unmittelbar ein nun auch expliziter und programmatischer „Abschied vom Zentrum“ (SŁAWIŃSKI 1994). Hinzu kam die Entideologisierung der angeblich „wiedergewonnenen Gebiete“ im Westen und Norden Polens, die während der kommunistischen Ära mit einem Identifikationsgebot (,uralt polnisches Land") überschrieben worden waren (vgl. z. B. ZYBURA 1999 und ŻYTINIEC 2007), dem die tägliche Wahrnehmung widersprach, ohne dass sie lange Zeit ausgedrückt oder ihr nachgegangen werden konnte. Bezeichnenderweise stammen die meisten der oben genannten polnischen Schriftsteller, die den ,regional turn“ der jüngeren polnischen Literatur verkörpern, aus diesen Gebieten oder leben dort.

In jedem Fall breitete der Nationalstaat oberhalb der Regionen einen größeren nationalen Raum aus, in dem die Regionen in weitestgehender Assimilation aufgehen sollten. Diese Formulierung ,breitete [...] oberhalb der Regionen einen größeren [...] Raum aus“ nun, und damit kommen wir zurück zum ,spatial turn“, ist nur möglich mit dem relativ gewordenen ,Raum'-Begriff des „spatial turn“. Denn natürlich kann nicht über ohnehin schon vorhandenen geographischen Räumen einfach so ein anderer Raum (woher sollte er denn kommen?) ausgebreitet werden. Man kann nicht Länder beliebig verschieben und Amerika, Papua-Neuguinea oder Atlantis über Ostpreußen, Grönland oder die Sahara legen. Offensichtlich meint ,Raum ' hier mehr und anderes als nur ein geographisches Gebiet, das am objektivsten mit den mathematisch exakten Angaben seiner Längen- und Breitengrade umschrieben wäre. ,Raum' im hier gebrauchten Sinne ist eine zur geographischen Erstreckung eines wie auch immer zu definierenden Gebietes hinzukommende semantische Dimension, quasi in Analogie zu den ,mental maps“ eine ,cultural map“ (JoACHIMSTHALER 2005:251), die den jeweiligen Raum mit Sinn auflädt, der aus der jeweiligen Gegend allein nicht kommen kann. Dies fängt schon an mit der oft willkürlichen Benennung von Gebieten: Dass bestimmte Flächen aus der Erd- 
oberfläche quasi herausgeschnitten und einen einheitlich gemeinsamen Namen bekommen, lässt sich (abgesehen vielleicht von Inseln und eindeutigen geologischen Formationen ${ }^{2}$ ) aus der Beschaffenheit dieser Flächen heraus kaum je begründen. Meist waren es zufällige politische oder administrative Konstellationen, die die Grenzen dieser Flächen festgelegt und ihnen irgendwelche Benennungen zugewiesen haben. Mit diesen Namen sind dann jedoch oft weitreichende Semantisierungen verbunden, „Amerika“ z.B. ist zwar als Benennung eines neu entdeckten Kontinents nach einem der Entdecker ein vergleichsweise einfallsloser Name, aber nunmehr doch längst keineswegs nur eine geographische Bezeichnung mehr, sondern eine, in die Unmaß an Hoffnung eingeflossen ist, utopischer Energie, Begehren (und schließlich auch Ablehnung, Neid und Hass). „Amerika“ bedeutet. „Amerika“ bedeutet mehr als einen geographischen Raum. Bezeichnungen wie „Sibirien“ oder „Mongolei“ können aufgrund ihres semantischen Bedeutungsüberschusses metaphorisch auf Gebiete angewandt werden, die rein geographisch mit den Regionen gar nichts zu tun haben, die diese Bezeichnung tragen - BRAKONIECKI (1998) schließlich kann im Zuge des polnischen „spatial turn“ Ostpreußen als „Atlantis des Nordens“ bezeichnen und damit zum Stichwortgeber für ein neues regionales Selbstbewusstsein werden. $\mathrm{Zu}$ unterscheiden ist also zwischen geographischen Räumen und Bedeutungsräumen bzw. „Sinnräumen“ (SCHMITZ 1993:418), die zu diesen hinzukommen und sie überlagern (können). In diesem Sinne wäre der Nationalstaat der Raum einer organisierten Bedeutung (,deutsche Nation“), mit der ein von ihm zu unterscheidendes Gebiet überschrieben wird.

Das Verhältnis zwischen scheinbar natürlich gegebenem geographischem Raum und menschlich hinzugefügtem „Sinnraum“ ist kein bloßes bezugsloses Nebeneinander. Der „Sinnraum“ und die beamteten Verwalter seines „Sinnes“ versuchen den geographischen Raum zu erfassen und dem Sinnraum anzupassen. Landschaftliche Erscheinungen werden deshalb ideologisiert (man denke nur an den „,deutschen Rhein“) ja, es wird sogar versucht, den geographischen Raum so umzuwandeln, dass er der Semantik des Sinnraumes soweit als möglich entspricht. Solche „Kulturraumformung“ (JOACHIMSTHALER 2002a) besteht nicht nur aus physischen Operationen, der Errichtung von Denkmälern und Bauwerken, Brücken und nach Herrschern benannten Straßen, also unmittelbar wahrnehmbaren Sinnmarkierungen in der Landschaft, sondern mehr noch in einer Kulturarbeit, die nicht nur die Landschaft, sondern auch ihre Re-

2 Doch selbst der Pfälzerwald trägt diesen Namen erst seit 1843, in welchem er auf einer Tagung von Forstleuten über neue Forstwirtschaftsregeln für die „Waldungen auf dem bunten Sandsteingebirge der Pfalz" vorgeschlagen wurde. 
zipienten, ihre Bewohner, Besucher und Betrachter (die im Medienzeitalter nicht mehr unbedingt selbst in die jeweilige Landschaft reisen müssen) in eine „Semiosphäre“ (der Begriff stammt von LOTMANN 1990, zum Begriffsgebrauch vgl. JOACHIMSTHALER 2005 und 2007) einzuhegen versucht. Raum verwandelt sich in Text, in Erzählung, Erklärung und Deutung des Raums. Semiosphären umgeben den Betrachter mit Zeichen- und Bedeutungsträgern, die ihn im Idealfall vollständig in das von diesen evozierte Weltbild einhüllen. Semiosphären sind halb physisch real (sie sind ja auf Bedeutungsträger und Zeichen angewiesen), halb mental (die Bedeutung der Zeichen muss im Bewusstsein der Rezipienten aktiviert werden). ,Raum'-Arbeit auf dieser Ebene ist deshalb nie Formung einer Landschaft allein, sondern immer auch (und oft in erster Linie) Formung von Menschen und Bewusstseinen. Der Nationalstaat des 19. Jhd.s (deshalb haben wir ihm so viel Platz einräumen müssen) ist der bis heute nachwirkende Idealfall der Etablierung einer Semiosphäre, in der sich Menschen wie in einem materiell realen Raum bewegen. Semiosphäre formt ihre Wahrnehmung und hat für jeden Sinneseindruck gleich die richtige (nationale) Interpretation bereit. Deshalb sind die Nationalstaaten Sinnräume, die sich über die kleineren Regionen gelegt haben. Lenkbare Kollektive werden möglich allein dadurch, dass ihre Mitglieder von derselben Semiosphäre umgeben werden.

,Raum der Nation' meint dabei zugleich das national erwünschte Territorium und die Art und Weise, wie dieses organisiert sein, wie in ihm gelebt werden soll. Der Nationalstaat besteht nicht nur aus der Beanspruchung eines Territoriums, sondern auch aus dessen organisatorischer Durchdringung und der Formung seiner Bewohner. Nichtadaptierbares, Fremdes, Anderes gehört im Normalfall nicht zur Imagination des, eigenen' Staates und bereitet deshalb so viele Schwierigkeiten, sobald es in der Wirklichkeit dann halt einfach dennoch da ist. Ungefragt und einfach nur so. Solche, nicht hierhergehörige ' Menschen stören dann den schönen Traum, die zart und sensibel in sich selbst ruhende, feinfühlige Phantasie von einer ungestört schönen Welt. Semiosphären haben immer einen irrealen, einen träumerischen Anteil. Je größer dieser ist, desto größer ist ihre Anziehungskraft. Vielleicht auch desto tödlicher. Mit ihrer Verführungskraft jedenfalls überlagerten die nationalen Semiosphären die regionalen Lebenswelten und mobilisierten die Menschen von ihnen hinweg in gröBere Vorstellungsräume hinein. Wo dann Nationalstaaten entstanden (oder sich expansiv ausbreiteten) und die imaginativen Semiosphären in fassbare und begehbare territoriale Wirklichkeit umzuwandeln versuchten, wurden die regionalen Lebenswelten realem Anpassungsdruck unterworfen. 
Im Zuge dieser Entwicklung wurde europaweit nahezu alles Wissen national perspektiviert: Nationalökonomie, Nationalgeschichte, Nationalliteratur, Nationalphilologie, nationales Recht, Nationalkultur, Nationalsprache usw. sind Wortprägungen, die zeigen, wie an sich anationale Wirklichkeitsbereiche dem neuen nationalen Paradigma unterworfen wurden und innerhalb der nationalen Semiosphären nur noch so weit Interesse fanden, als sie mit nationalen oder als national interpretierbaren Teilen in diese hineinreichten. Nationaler Monomonismus verwandelte die Wissenschaften in eine Reihe halbblinder Nationalwahrnehmungen, die bestätigten, dass das wenige, was sie noch wahrzunehmen erlaubten, tatsächlich dem Wahrnehmungskriterium national entsprach. Emanuel Geibel war ein deutscher Schriftsteller, Heines Zugehörigkeit zur deutschen Nationalliteratur hingegen konnte bestritten, seine Beachtung im nationalen Gedächtnis kritisiert und so weit als möglich unterbunden werden (PeTERS 1990). Die nationale Perspektive erlaubte Einschluss- und Ausschlussverfahren nach Maßgabe der jeweiligen (durchaus variablen und veränderlichen) Definition von ,national'.

Die wichtigsten Hilfswissenschaften der Nationalisierung, der Einbindung der Bevölkerung in den Nationalstaat, den nationalen ,Raum“ waren die Philologien, genauer: die neu entstehenden Nationalphilologien. Sie bildeten die für die Einführung der Bevölkerung in nationales Denken und Fühlen unverzichtbaren Muttersprachlehrer bzw. deren Ausbilder aus und formten das im muttersprachlichen Unterricht vermittelte nationale Weltbild. Die Konstitution des Gegenstandsbereiches der Nationalphilologien war zugleich die Konstitution der nationalen Semiosphäre. Sie konstruierten die Nation und ihr Selbstbild aus „Zeugnissen der Vergangenheit“, einer „Vorgeschichte“, in der die Nation bereits einmal so sehr sie selbst gewesen sein sollte, dass man dieses ihr nur wiederzuerweckendes ,eigentliches“ Wesen einer (noch) ungenügend nationalisierten Gegenwart wie ein befreiendes Dispositiv für kollektiv „eigene“ Identitätsbildungen entgegensetzen konnte. Die Nationalphilologien siebten aus den Zeugnissen der Vergangenheit alles als national Interpretierbare aus und erfanden der entstehenden Nation nationale Traditionen, die Gebrüder Grimm wollten den Deutschen eine Vergangenheit als die ,ihrige“ so „nah“ (GRIMM 1865:5) wie möglich bringen, den „,vergangenen Zuständen“ sollte eine ihnen bisher fehlende „Bedeutung für die Gegenwart“ zugeschrieben werden (UHLAND 1865:17).

Die nationale Entwicklung der Literaturgeschichte seit dem 18. Jhd. (WEIMAR 2003) bedeutete aber (mit Wirkungen bis heute) zugleich auch die zunehmende Umwandlung des bis dahin selbstverständlichen literarischen Unter- 
richts, also aktiv betriebener Rhetorik und Poetik zum Zwecke gelehrter produzierender Ausdrucksfähigkeit in ein bald vorwiegend passives, rezipierendes nationales Wissen, das letzten Endes an die Stelle selbständigen Könnens Überzeugung setzte. Die Einführung der allgemeinen Schulpflicht verwandelte die bisher einer kleinen Elite vorbehaltene Ausbildung in praktisch anwendbaren Kulturtechniken in eine Unterrichtung breiter Massen, denen aus machtpolitischem Kalkül - nicht mehr beigebracht wurde, selbst aktiv zu schreiben und zu formulieren, sondern ihre eigene Unfähigkeit dazu einzusehen (GRIMBERG 1988). Sie sollten bewundernd aufschauen zu einer Elite, der sie selbst nicht angehören sollten - und in deren literarischen Produkten sie doch zugleich ihr national ,Eigenes" in Akten identifikatorischer Verehrung wiedererkennen können sollten. An die Stelle einer Pädagogik des eigenen Tuns trat die Didaktik des Auswendiglernens national bedeutsamer Texte. Die Systematik der Textsorten und Stilmittel wurde aufgebrochen, die bis dahin tradierten Textbeispiele (vorrangig aus der Antike) wurden ausgetauscht, durch nationale ersetzt und im Sinne einer behaupteten Entwicklung der Nation zum nationalen Höhepunkt chronologisch angeordnet, so dass als Ergebnis literaturwissenschaftlicher Darstellung schließlich nicht mehr die technische Erklärung gelungenen Schreibens galt, sondern die Genealogie zu verehrend ,heiliger', weil nationaler Texte. ${ }^{3}$ Selbst der noch aus vornationaler Zeit stammende altphilologische Unterricht (der jetzt oft mit nationaler Argumentation bekämpft wurde) beruhte nun oft auf einer nationalisierenden Interpretation der Antike. Jede Semiosphäre schafft sich ihre eigene Kultur und passt die Kulturtechniken der Notwendigkeit an, die Semiosphäre durch Ausbildung ,gläubiger' Schüler aufrechtzuerhalten. Glauben wird zur wichtigsten Tätigkeit, die darin besteht, das, woran geglaubt werden soll, durch Glauben im Glauben zu

3 Damit sei, um auf einen Einwand von Maria Kłańska zu reagieren, nicht behauptet, dass in vornationaler Zeit Unterricht und Lehrer prinzipiell besser gewesen wären. Natürlich konnte auch der Latein- und Griechischunterricht mit seinem Zwang zur Vokabel- und Grammatik-Paukerei eine Qual sein. Aber zum einen herrschte noch kein allgemeiner Schulzwang mit national zunehmend vereinheitlichten Lehrplänen für kulturpolitisch als formbar betrachtete Massen, zum anderen sollte die Ausbildung nicht in eine nationale, sondern in eine konfessionelle Semiosphäre einpassen, innerhalb derer die Schüler dann als Geistliche Führungsfunktionen übernehmen sollten, deren Ausübung die Fähigkeit rhetorisch wirkungsvollen Ausdrucks (etwa zum Zwecke der Predigt) verlangte. Solch ,elitäre Ausbildung gab es natürlich auch noch an den Gymnasien des 19. und 20. Jhd.s sie war nun aber nicht mehr der Normalfall schulischer Ausbildung. 
Jürgen Joachimsthaler

erhalten. Es ist wie bei jeder Religion: Glaube ist als Ausbildungsziel wichtiger als Können.

Absurdes, bis heute nachwirkendes Ergebnis dieses Umgangs mit Literatur war, dass das Wie des Verfasstseins behandelter Texte (incl. der Diskussion technischer Alternativen) immer weniger Gegenstand des Unterrichts sein durfte, weil sie ja zu ,heilig' waren, als dass man mit ihrem Textbestand experimentierend eigene Erfahrung hätte machen dürfen. Goethe umschreiben? Auch nur versuchsweise? Das würde vielleicht helfen, gerade im Vergleich mit eigenen Umschreibungsversuchen seine (Goethes) Besonderheit und technische Qualität besser zu verstehen, wäre aber als ein Akt der Blasphemie im nationalen Unterricht undenkbar. Die Nationalphilologien etablierten einen nationalen Kanon und mit ihm eine nationale Hülle aus unberührbar quasisakralen Texten, die das einzelne Mitglied der Nation möglichst fugenlos in die von ihnen verbreiteten Weltbilder einbinden sollte. Da aber natürlich auch ,heilige" Texte falsch verstanden werden können, musste an die Stelle der Text-Analyse noch eine zu erlernende, richtige ' Interpretation treten, die als Leseanleitung für ,richtige' Lektüre sorgen sollte (Goethe hätte sich sicher über die nationalen Faust-Interpretationen sehr gewundert). Literaturwissenschaft wandelte sich von der Kunst des Schreibens und schreibkundigen Lesens in die Anleitung semiosphärenkonformen Lesens.

Die Nationalphilologien bauten damit einen vorrangig aus Texten bestehenden nationalen Tempel auf. Als dessen unterirdische Krypta, als dessen , heiliger Boden' diente die Geschichte; der ,Quell' der Überlieferung kam nun zum ,Raum‘, zum Territorium der Nation als eine in dieser Form bis dahin unbekannte historische Tiefendimension und Sinngeschichte hinzu. Die Semiosphären sollten ja die Semiosphärenbewohner von möglichst allen Seiten umgeben, Blick und Denken der Bewohner sollten, wohin immer sie streiften, auf Semiosphäreninnenwände stoßen, die Wahrnehmung oder auch nur Bedenken eines jeden Außerhalb so weit als möglich unterbanden. ${ }^{4}$ Die literaturhistorische Konzentration auf die Geschichte diente dabei nicht nur der Rekonstruktion angeblicher nationaler Eigenart aus ihrer Vorgeschichte, sondern auch der Abdichtung der Semiosphäre gleichsam nach , unten', der Vergangenheit zu. Auch dort durfte nachfragendes Denken keine andere Antwort auf seine Fragen erhalten als eine nationale. Ein nicht-nationaler Ursprung der Nation sollte

$4 \quad$ In archivierten Schulbeständen aus dem Kaiserreich fand ich eine am 25.10.1911 erneuerte Regierungsanordnung vom 11.12.1905, die Lehrern, die im Ausland gewesen waren, untersagte, darüber zu berichten: ARCHIWUM PAŃsTwOwE W OPOLU: Kreisschulinspektion Carlsruhe 53, S. 56. 
undenkbar sein. Nichts ist ja heikler als die Frage nach der Herkunft der Nation. Kommt sie aus dem Nichts? Oder stammt sie von jenen Anderen, von denen man sie abzugrenzen versucht? Nationalgeschichte sucht deshalb nationale oder national verträgliche Ursprungsgeschichte(n) für die jeweilige nationale Semiosphäre zu rekonstruieren und der nationalen Semiosphäre zu unterlegen. Nationalphilologie stattet diese Geschichte dann mit Texten aus, mit Geschichten, Vorstellungen, Phantasie, ,tapeziert' sie sozusagen, macht sie wohnlich. Aus diesen Gründen auch stieg deutsche Sprachgeschichte bis in vorgermanische Zeiten zurück und mühte sich, Sprachstände als ,indogermanisch' zu rekonstruieren, aus denen der deutsche als deren legitimster Erbe quasi natürlich hervorgewachsen sei (während in anderen Ländern neutraler von einer ,indoeuropäischen' Sprachstufe gesprochen wurde). Die Nationalphilologien waren so integraler Bestandteil der nationalen Semiosphären und entscheidend an deren Entstehung und schließlicher Verfasstheit beteiligt. Sie schlossen aus, was nicht gefiel, und homogenisierten den Rest zu einer in sich widerspruchsfrei wirkenden intellektuellen Legierung um Wissens- und Vorstellungsbestände, mit denen die Semiosphärenbewohner umgeben, in die sie eingeschlossen wurden.

Von hier aus lässt sich nun erklären, warum im „spatial turn“ überhaupt von einer „Wiederkehr des Raumes“ die Rede sein kann, als ob dieser für einige Zeit verschwunden gewesen wäre (sonst könnte er ja nicht „wiederkehren“). Natürlich war nicht „,der Raum“ verschwunden - Kolonialisierung und Nationalisierung waren sogar sehr raumbezogene Entwicklungen, die die Landkarten der Welt neu aufteilten, doch waren sie zugleich Bewegungen, die in ihrer Tendenz, den Raum umzuwandeln und ihrer jeweiligen Semiosphäre anzupassen, vom realen Raum (und seinen Bewohnern) mehr und mehr absahen und diese als bloße Masse der Kulturraumformung betrachteten. Was nicht zum Konzept passte, durfte nicht in den Semiosphären vorkommen (und wurde nicht selten sogar physisch real entfernt). ,Regionaler' Widerstand gegen solche Zurichtung war denn auch immer Widerstand gegen Kulturraumformung, gegen semiosphärische Assimilationspolitik. ${ }^{5}$

5 Das heißt nicht, aber das ist ein anderes Problem, dass regionale Bewegungen nach ,unten ' nicht ihrerseits sehr ähnlich operieren können, wie ja auch der Nationalstaat selbst eine ,Region', ein Gebiet darstellt, das gegenüber der universalen Globalisierung ebenso partikular ist wie ihm gegenüber ,seine‘ Regionen. Der Begriff ,Region' ist - in dieser Hinsicht - ein relativer und relationaler, der nur für die Zwecke dieses Aufsatzes zum Gegenbegriff zu ,Nation“ stabilisiert wird, ansonsten aber vielfältigere Anwendungsmöglichkeiten hat und selbstverständlich 
Die vom „spatial turn“ proklamierte „Wiederentdeckung des Raumes“ nun bedeutet erst einmal nur, dass gegen die Wahrnehmungsvorgaben der Semiosphären der Blick auf das gerichtet wird, was im jeweiligen Territorium überhaupt vorhanden ist. Die häufig (aus der regionalistischen Teilströmung des „spatial turn“) zu hörende Erklärung, die „Wiederentdeckung“ des Raumes komme einer Wiederentdeckung außerkultureller und -textueller Wirklichkeits- und Referenzebenen gleich, umschreibt in all ihrer wahrnehmungsrealistischen Naivität doch treffend den Vorgang einer Infragestellung herrschender Wahrnehmungsschablonen. Im Prinzip jedoch bedeutet dies zunächst nichts anderes als die Aufforderung, die räumliche (und strukturelle) Verfasstheit der Semiosphären und damit der Nationalstaaten zu reflektieren auf das hin, was ihre imaginäre Geographie und ihr Selbstverständnis aus sich ausschließen. Deshalb ist, weil Semiosphären als Bewusstseinsräume nicht so einfach verlassen werden können wie reale Räume durch die nächste Tür, selbstreflexive Infragestellung der eigenen Vorstellungsmuster so wichtig - der relative ,Raum“-Begriff des ,postmodernen“ Flügels des „spatial turn“, all die „,mental maps“ und „Semiosphären“, verdanken sich solch analytischer Taktik. Raumreflexion wird ihr eigenes Objekt, richtet sich gegen sich selbst und analysiert sich selbst. Semiosphären bilden mentale Umgebungen, ,cultural maps“, in die die meisten Menschen eher hineinwachsen, als dass sie an deren Gestaltung aktiv mitzuwirken Gelegenheit hätten. Man weiß nicht und fragt erst einmal gar nicht, warum man für richtig hält, was man zu wissen glaubt. Die Denke hält sich für wahr. Wir leben in der Selbstverständlichkeit dessen, was uns als die einzige Wirklichkeit erscheint. Dies erklärt so rätselhafte Erscheinungen wie mangelndes Einfühlungsvermögen und die unterdrückte Empathie nicht oder nicht vollständig wahrgenommenen Mitmenschen gegenüber ebenso wie die Grenzen des eigenen Vorstellungsvermögens insgesamt. Der Mensch endet, wo seine Phantasie endet, die wiederum häufig genug dort endet, wo seine Semiosphäre endet. Semiosphären schließen ein.

Und das gilt natürlich auch für jene nationalen Wissenschaften, die ,ihre` gesamte Welt auf die nationalen Semiosphären hin zu perspektivieren hatten. Um die Grenzen des Nationalen in den Nationalwissenschaften, in den Nationalphilologien auszutasten, gilt es deshalb, den nationalen Blick auf sich selbst zurückzurichten, also zu fragen, was warum überhaupt als national galt. Dies

auch jeden Nationalstaat, jede postmoderne Metropole, jede Sternenhäufung in unserer Galaxis und jede Galaxienhäufung im Universum, jedes Paradies und jede Hölle bezeichnen kann. All das sind ja ,Regionen', Orte, Häfen oder Wüsten. Irgendjemandes ,Heimat', irgendjemandes Gefängnis. 
ist nun auf den ersten Blick natürlich ein Schritt, der in Deutschland und der Germanistik nach dem national(sozial)istischen Zusammenbruch bereits vollzogen worden zu sein scheint. Niemand würde heute mehr die Zugehörigkeit Heines zur deutschen Literaturgeschichte bestreiten, die Frauenbewegung hat eine Öffnung des Kanons und der Wahrnehmung für in der germanistischen Literaturwissenschaft zuvor zu wenig berücksichtigte weibliche Literatur erfochten. Der nationale Kanon ist in seiner Fragwürdigkeit durchsichtig geworden und seither flexibel bis zur Unschärfe - und doch bleibt er ein nationaler. Man hat den Bereich dessen, was im Fach berücksichtigt wird, erweitert, nicht aber die Grundlage der Auswahl selbst reflektiert. Die Nationalphilologie denkt über sich nach, bleibt aber Nationalphilologie (und als solche unglücklich über sich selbst bis hin zur Inszenierung einer unaufhörlichen „Krise der Germanistik").

Der „spatial turn“ hält die dazu passende Frage, eine auf den ersten Blick sehr einfach wirkende Frage bereit. Sie lautet nicht: Wie soll ,Deutsche Literatur', wie soll nationale Literatur überhaupt definiert werden, wann soll ein Text als zu ihr gehörig betrachtet werden und wann nicht? Diese Frage kann nur national- und entwicklungslogisch beantwortet worden. Die auch heute im Fach noch gültige Definition seines Gegenstandsbereiches lautet unausgesprochen: Für uns ist ein Text relevant, wenn er der nationalen Semiosphäre zugehört und innerhalb der Entwicklung derselben eine wie auch immer geartete Rolle spielt. Dies kann - so der Normalfall - durch Nationalsprachigkeit des Textes (eine im historischen und globalen Vergleich keineswegs selbstverständliche Eigenschaft) begründet sein oder durch die Thematik eines Textes (dadurch kann ein französischsprachiges Werk - DE STAËL 1813 - Teil der deutschen Literaturgeschichte werden), vielleicht auch durch eine als national relevant erscheinende Herkunft des Autors (dadurch gerät der englischsprachige Schriftsteller Joseph Conrad in die polnische Literaturgeschichte) - es können aber auch Texte als national bedeutsam vorgestellt werden, die nun wirklich nichts mit der Nation zu tun haben, sondern nur als für den imaginierten $\mathrm{Zu}-$ stand der Nation vorbildliche Beispiele aus anderen Nationalliteraturen dienen (man denke an die Rolle Ossians und Shakespeares in Herders für die Entstehung eines deutschen Nationalbewusstseins so bedeutsamen Textsammlung Von deutscher Art und Kunst - [[HERDER] 1773] -, in der kein einziger Aufsatz einem deutschsprachigen Autor gewidmet ist). Wenn aber schon die scheinbar so eindeutige Zuordnung zur nationalen Literatur so willkürlich ist was wissen wir dann wirklich darüber, was aus ihr ausgeschlossen worden ist? 
Der „spatial turn“ lädt nun dazu ein, die Frage nach dem Gegenstand der Philologien neu zu beantworten, nämlich nach einem ziemlich einfachen räumlichen Kriterium: Gegenstand der Germanistik sollte dann nicht mehr eine wie auch immer zu definierende, deutsche' oder nationale Literatur sein, sondern schlicht und einfach „Literatur in der Region“ (HARTMANN 2002). Diese kann, wenn man denn am Attribut, deutsch ' unbedingt festhalten will, die in Deutschland (oder den deutschsprachigen Ländern) geschriebene Literatur sein, erweiterbar je nach Ansatz und Bedürfnis um solche Literatur, die in Deutschland gelesen und nachgeahmt oder bekämpft wurde (so behielte Shakespeare seine Rolle) oder in sonst einem Bezug zu Deutschland stünde, sei es als Literatur von Emigranten, sei es als Reiseliteratur über Deutschland (dann würde Madame de Staël ihren Platz erhalten können). An die Stelle eines entwicklungslogisch kausalisierten national bewegten ,Nacheinander" würde dann ein räumliches ,Nebeneinander ' des in der Region gleichzeitig Anwesenden treten, synchrone Schnitte durch den Ablauf der Literaturgeschichte also ein Übergewicht über die Epochenfolge bekommen. Auf den ersten Blick wäre dies nur eine geringe Akzentverschiebung: Auch jetzt schon gilt es ja, Synchronie und Diachronie in Überblickswerken auszubalancieren. Diese Aufgabe würde nicht verschwinden, auch die synchronen Schnitte müssten in ihrer zeitlichen Abfolge irgendwie miteinander verbunden werden.

Ein solcher räumlicher Blick auf das im jeweiligen Territorium gleichzeitig neben- und miteinander Anwesende würde jedoch, und darum geht es eigentlich, schnell zu der Erkenntnis führen, dass es in Deutschland, wenn man denn an dieser Region (vorerst) festhalten will, neben der deutschsprachigen immer auch schon anderssprachige Literatur (mit den dazugehörigen Institutionen, Verlagen, literarischen Kreisen etc. ${ }^{6}$ ) gegeben hat. Gehen wir einmal nur von den Grenzen Deutschlands nach 1945 aus (auf die Thematisierung der innerdeutschen Grenze verzichte ich), so zeigt sich bei nicht national beschränktem Hinsehen schnell, dass es neben der deutschsprachigen Literatur auch eine sorbischsprachige gibt, die mit dem erst kürzlich verstorbenen Jurij Brězan sogar einen Autor von Weltformat hervorgebracht hat, der dennoch - und obwohl er sogar etliche Werke, darunter seinen wichtigsten und bedeutendsten

6 HEYDEBRAND (1999:14) ist in ihrer methodologischen Grundlegung der Untersuchung literarischer Regionen nicht an unserer Fragestellung nach der Einbeziehbarkeit kulturell bzw. semiosphärisch , anderer' Literaturen ausgerichtet, lässt sich aber problemlos dafür fruchtbar machen und damit vereinen: „,Literaturregionen“ [...] lassen sich am besten von Orten und Institutionen literarischer Kommunikation her erfassen." 
Roman (BRĚZAN 1976), auf deutsch geschrieben hat - nicht als Bestandteil der Literatur Deutschlands im 20. Jhd. betrachtet wird, weil die Sorben, mögen sie auch Staatsbürger des jeweiligen deutschsprachigen Staates sein, der ihr Sprachgebiet mit einschließt, unter den nach wie vor gültigen Bedingungen der Definition der Nation eben nicht als ,deutsch ' gelten und nie ,Mitglied ' der deutschen Semiosphäre waren. „Die sorbische Literatur [...] ist [...] - weitestgehend unbemerkt von der deutschsprachigen Öffentlichkeit - zu einer neuen deutschen Literatur herangewachsen." (KoschmaL 1993:299) Der Slawist Koschmal gebraucht ,deutsche Literatur" hier im räumlichen Sinne einer ,Literatur in Deutschland'. Eine solche Literatur aber hat in der (deutschen) Germanistik bisher keinen Platz. Brězans Enttäuschung ist nachvollziehbar: „Daß Dissertationen zu Krabat in Litauen, Rußland, Polen und auch hier im Lande verfaßt wurden, tröstete mich wenig darüber, daß das Buch in der literarischen Öffentlichkeit der Bundesrepublik offenbar als nicht erschienen galt." (BRĚZAN 1999:211)

Offensichtlich hat die Nation - und mit ihr ,ihre` Nationalphilologie - den Raum und die in ihm lebenden Menschen und ihre Literaturen so sehr mit ihrer Semiosphäre überdeckt, dass kein Ort mehr für die Wahrnehmung von etwas übrig blieb, das nicht so recht mehr als ,deutsch', als national klassifiziert werden konnte (wiewohl die Texte der sorbischen Literatur selbst teilweise deutschsprachig sind). Betrachtet man nun vollends die Geschichte der Literatur in dem jeweils „Deutschland“ genannten Territorium auch vergangener Zeiten, so fällt auf, dass es in der deutschen Literaturgeschichte keinen Platz gibt auch nur für die Thematisierung der Tatsache, dass in Oberschlesien (vgl. als große, nie in den allgemeinen literaturgeschichtlichen Umlauf aufgenommene Ausnahme LuBOs 1974:474-655), Westpreußen (vgl. etwa DERDOWSKI 1880), den polnischen Teilungsgebieten Preußens (vgl. KRZYŻANOwSKI / HERNAS 1985:226-228), Masuren (vgl. KĘTRZYŃSKI 1938) und in Kleinlitauen (vgl. JOACHIMSTHALER o.J.) eine jeweils nicht deutschsprachige Literatur überhaupt existiert hat (von den Sorben ganz zu schweigen). Ähnlich ließe sich die Frage nach der Berücksichtigung auf polnischem Boden entstandener jiddischer, ukrainischer oder deutschsprachiger Literatur stellen.

Das Problem rührt ans Selbstverständnis der Nationalphilologien: Diese gingen von einer Gegenstandsbestimmung aus, die vorstrukturiert war durch eine nationale Semiosphäre und deshalb die Berücksichtigung anderer Semiosphären unnötig machte. Zugleich wurden diese nationalen Semiosphären so auf den realen Raum projiziert, dass in diesem wie in der Semiosphäre selbst nichts zu existieren schien, was nicht zur Semiosphäre hinzugehörte. Der Zorn, 
den das Auftreten unerwarteter (weil bisher nicht beachteter) nationaler Minderheiten in einigen sich für (national), gebildet ' haltenden Kreisen auslösen kann, rührt gerade hierher: Es (das Unerwartete) widerspricht einem ganzen Kultur- und Lebenskonzept. Dieses ist so wirksam, dass es aus Sicht der Nationalphilologie sehr schwierig ist, überhaupt irgendwie mit gleichzeitig im gleichen Raum befindlichen ,anderen“ Literaturen umzugehen. Ein schönes Beispiel hierfür ist die aktuelle Welle interkultureller Literatur, an die sich mittlerweile auch eine eigene „Interkulturelle Literaturwissenschaft“ innerhalb der Germanistik anschließt (CHIELLINO 2000; HoFMANN 2006). Diese konzentriert sich auf Literatur, die interkulturelle Begegnung zum Gegenstand hat, Kulturen also tendenziell als voneinander getrennte Blöcke behandelt, die sich über klar voneinander abgegrenzte unterschiedliche Territorien erstrecken und dann in interkulturellen Kontakt miteinander geraten, wenn Individuen aus einer Kultur die Grenze zur anderen Kultur überschreiten. Gegenstand der Interkulturellen Literaturwissenschaft sind so vorrangig Reise- und Migrationsliteratur. ${ }^{7}$ Damit freilich wird an der Überschreibung der Territorien durch nationale Semiosphären festgehalten und das eigentlich interkulturelle Problem, eine nicht durch Mobilität zum Raum hinzukommende, sondern durch Unterschiedlichkeit der Bewohner des Raumes immer schon vorhandene Polykulturalität innerhalb der Territorien selbst ausgeblendet. Die Sorben oder die Polen in den Teilungsgebieten wanderten nicht nach Deutschland ein, sie waren, will man denn noch immer und schon wieder in solchen Kategorien denken, immer schon da - lange vor den Deutschen. Und auch viele der Autoren sogenannter „Migrantenliteratur" sind in Deutschland geboren und aufgewachsen und wehren sich verständlicherweise gegen das Etikett „Migrant“. Aus nationalsemiosphärischer Sicht jedoch kann man sie kaum anders bezeichnen: Für den Bewohner der Nationalsemiosphäre sind sie, wenn sie denn überhaupt in seine Wahrnehmung geraten, unversehens Hinzukommende von außerhalb der Semiosphäre. Das Problem besteht nur darin, das Außerhalb der Semiosphäre gleichzusetzen mit einem Außerhalb des Territoriums, auf das die Semiosphäre sich nach wie vor so projiziert, als wäre sie mit ihm identisch. Dann erscheinen diese „Migranten“ nach wie vor als Zuwanderer minderen Rechts und die Sorben als nicht vorhanden.

7 HofMANN (2006:121-130) widmet immerhin ein Kapitel auch der Blechtrommel von Günter Grass (1959) und der darin dargestellten kulturellen Hybridität Danzigs - er braucht Grass, weil sich Salman Rushdie, einer der Schlüsselautoren Hofmanns, auf ihn beruft (RUSHDIE 1990:175; HofMANN 2006:123f., 184-194). 
Doch tatsächlich gibt es in einem Territorium selten nur eine Semiosphäre. Oft überlagern in ihm sich die Semiosphären, ohne dass deren Bewohner einander wechselseitig wirklich wahrnehmen müssten. Sie leben in voneinander geschiedenen Parallelwelten und Semiosphären und reagieren auf im selben Raum anwesende Zeichen nur dann, wenn diese eine Bedeutung für die ,eigene' Semiosphäre haben (ein bewusst exotisierendes Beispiel: Am selben Ort können Buddhisten und Hinduisten leben. Doch ihr Leben ist an unterschiedlichen Tempeln und den von diesen ausgehenden Signalen ausgerichtet. Der Hindu reagiert normalerweise ebenso wenig auf ein buddhistisches Signal wie umgekehrt. Man ist gewöhnt, dass die jeweils ,anderen' Signale da sind und hört und sieht sie nicht mehr. Sie sind „Rauschen“). Ich habe dieses Problem einmal als „Kulturraumverdichtung“ zu umschreiben versucht (JOACHIMSTHALER 2002a): Kulturräume sind variable, fluktuierende Größen, die mit Menschen, Büchern und oft auch nur Dingen sich ausbreiten können. Es gibt einen katholischen (und einen buddhistischen, einen chassidischen, einen lutherischen etc.) Kulturraum und einen Kulturraum der deutschen Sprache (innerhalb dessen wiederum verschiedene Kulturräume und verschiedene Dialekte existieren), aber auch die Kulturräume aller anderen Sprachen, einen Kulturraum der deutschen Verwaltung, einen Kulturraum des Bieres und einen Kulturraum des Kartoffelanbaus, einen Kulturraum von McDonalds und einen Kulturraum des Schachspiels etc. etc. Kulturräume sind Verbreitungsräume jeweils eines Kulturgutes. Es gibt keinen Menschen und keinen von Menschen bewohnten Raum, keine ,Region', die nicht eine Überschneidung zahlreicher Kulturräume wäre. Dabei überschneiden sich einerseits kategorial unterschiedliche Kulturräume wie z.B. der eines Getränkes, der einer Konfession, der eines Staates und der einer Sprache, gleichzeitig aber überschneiden sich auch innerhalb der Kategorien miteinander verwandte und oft konkurrierende Kulturräume: Es gibt wohl keinen Ort auf der Welt, an dem es nicht verschiedene Getränke gibt, an dem sich also nicht die Kulturräume verschiedener Getränke überschneiden.

Meist sind nur die staatlich-administrativen Kulturräume wirklich territorial eindeutig voneinander abgegrenzt und versuchen dann, innerhalb ,ihres' Territoriums eine Semiosphäre zu erzeugen, um die Bevölkerung stärker an sich zu binden und zu durchformen. Zu diesem Zweck werden verschiedene der auf dem Staatsgebiet sich überschneidenden Kulturräume zu einer Kulturraumverdichtung gebündelt, die dann das in der Semiosphäre vorherrschende Identitätsmuster abgeben sollen, indem sie z.B. eine Sprache mit einer Konfession und einer Ethik und vielleicht sogar einer Kleiderordnung zum für gültig erklärten Identitätsmuster zu verdichten suchen. Dass es im gleichen Territorium 
gleichzeitig auch noch Sprecher anderer Sprachen, Gläubige anderer Konfessionen etc. gibt, wird marginalisiert wo nicht gar bekämpft. Dadurch erst entsteht der Eindruck, es gäbe z.B. ein ,Deutschland', dem (und dessen Bewohnern) erkennbare , deutsche ' Eigenschaften zugeschrieben werden können, die das Land von anderen Ländern unterscheidet. Doch faktisch gibt es im Kulturraum des Bieres häufig auch Weintrinker und umgekehrt. Die Kulturräume überschneiden sich. Und dies gilt auch für Konfessionen und Sprachen, ,Nationalitäten“.

Der „spatial turn“ ermöglicht es nun durchaus, die Literatur eines, z.B. des katholischen oder des deutschsprachigen Kulturraumes (oder auch des zu einer bestimmten Zeit „Deutschland“ genannten Territoriums) zu beschreiben, aber er verlangt, dass jede mit dieser Literatur und ,ihrem ' Kulturraum verbundene semiosphärische Konstruktion mit reflektiert wird. Doch der eigentliche Reiz des ,spatial turn“ liegt in seiner Möglichkeit, völlig Abschied zu nehmen vom nationalen Paradigma und auf der Gegenstandsebene Räume neu zu konstituieren, innerhalb derer dann das Neben- und Miteinander aller dort vorhandenen Literaturen untersucht wird. Ein solcher Raum kann immer noch „Deutschland“ heißen, aber auch „Mitteleuropa“ (KONSTANTINOVIĆ / RINNER 2003), „Galizien“ (KASZYŃSKI 1987) oder „Breslau“ (GRASZEWICZ / ZYBURA 1997). Der jeweilige Raum braucht nicht mehr national definiert zu werden. Interkulturalität und zwischenkulturelles Mit- und auch Gegeneinander lassen sich, so die Erkenntnis, nicht von der nur immer auf die eigene Semiosphäre konzentrierten Nationalphilologie aus erfassen, sondern nur durch den Blick auf das Miteinander in konkreten Regionen (vgl. bereits STRUTZ 1992). An die Stelle der Chronologisierung einer ganz mit der Darstellung der nationalen Entwicklungsgeschichte beschäftigten nationalen Literaturgeschichte tritt eine auf das räumliche Miteinander des Verschiedenen konzentrierte interkulturelle Komparatistik (die sogar wieder anfangen könnte, Texte in poetischer Hinsicht miteinander zu vergleichen und intertextuell - und eines Tages vielleicht sogar wieder literarisch - mit ihnen zu spielen). Poesie tritt wieder an die Stelle der Nation, Raum, also räumliches Nebeneinander löst Zeit, also zeitliches Nacheinander als Gestaltungsvorgabe ab. An die Stelle des nationalen Entwicklungsromans und der analog dazu aufgebauten nationalen Literaturgeschichte mit der einen Zentralperspektive tritt der polyphon und auf viele Stimmen und Schauplätze verteilte, postmoderne' Roman mit seiner dekonstruktiven Ästhetik mit ihren gleichberechtigt losen Erzählfäden.

Deutlich sichtbar wird diese Ästhetik mittlerweile auch in literaturwissenschaftlicher Arbeit an der zunehmenden Zahl räumlich orientierter Antholo- 
gien (HEYDEBRAND 1999) und lexikonartig aufgebauter Darstellungen, die versuchen, verschiedensprachige Literaturen aus demselben Raum, aus derselben Region, demselben Territorium gleichberechtigt nebeneinander zu stellen (GRASZEWICZ / ZYBURA 1997; GRZELAK 2001; BRAKONIECKI / LIEPSCHER 2001; SCHMITZ / UDOLPH 2001) und damit nicht mehr Nationen, sondern interkulturelle Regionen und Orte mit Hilfe einer Poetik des Neben- und Miteinander zu evozieren. Die Atlantis des Nordens (BRAKONIECKI 1998) oder das ,postmodern' synkretistisch deutsche und polnische Traditionen miteinander verschmelzende Bresław (ZAWADA 1996) sind Beispiele für eine hybridisierende Utopie und die einander überlagernden Zeiten nebeneinander legende neue ,terra recognita“ (DZIKOWSKA 1997).

Nicht umsonst stammen viele dieser Bücher aus Polen, ging dort doch der „spatial turn“ einher mit einer Entdeckung der multikulturellen Traditionen der jeweiligen Region. Während in Deutschland auf oft gegenstandsferner abstrakter Ebene noch über die Sinnhaftigkeit und Verwendbarkeit dieses „turns“ in den Philologien diskutiert wird, sehen wir hier Ergebnisse, die das öffentliche Bild ganzer Städte und Landschaften mitformen und langsam verändern. Und mit ihnen die Textästhetik sowohl der Literatur als auch literaturwissenschaftlicher Darstellungen. Die Darbietungsweise in Anthologien (und Lexika) erlaubt es, Texten und Autoren im Nebeneinander ihr jeweiliges So-Sein zu belassen, ohne sie in eine ideologisierte (z.B. nationale) Gesamtnarration einbinden und die oft gegenstandsfremde Frage stellen zu müssen, wo sie denn nun, als wären sie nicht dort, wo sie sind, eigentlich ,hingehören ' (man denke nur an den Umgang mit Autoren jüdischer Herkunft in manchen nationalen Literaturgeschichten). An die Stelle einer national eingeschränkten Wahrnehmung tritt damit europäisch offene Weite und Großzügigkeit.

Gründete das nationale Paradigma auf binären Alteritätskonstruktionen, denen zufolge ein ,Eigenes' gegenüber einem ,Fremden' zu behaupten sei, so ermöglicht es das regionale nun, an einem Ort, in einer Stadt, in einer Region und in einer Landschaft räumlich neben-, mit- und durcheinander anwesende Kulturen, Bevölkerungen, Sprachen, Literaturen, Konfessionen, Traditionen etc. in ihrer Gleichzeitigkeit zu betrachten, statt sie weiterhin rhetorisch gegeneinander aufzustellen. Als Forschungsgegenstand von Interesse ist jetzt, was früher mit wissenschaftlichen Mitteln geleugnet, bekämpft oder zumindest (etwa in Form rein nationaler Literaturgeschichtsschreibung) ausgeblendet wurde, wie nämlich lokal einander überlagernde kulturelle Sphären unterschiedlicher Provenienz zueinander sich verhalten, sich vermischen, gegenseitig beeinflussen, einander anziehen und abstoßen oder gar in Konflikt miteinander geraten, nicht mehr aber, dass die Rechtmäßigkeit der Anwesenheit der einen am jeweiligen Ort gegen die der anderen ausgespielt wird. [...] Als neue Bezugsgrößen der Forschung treten an die Stelle der Nationen deshalb nun konkrete Orte oder Regionen, die jeweils exemplarische 
Jürgen Joachimsthaler

Funktion haben sollen für ein multiethnisches Europa und vor dessen Hintergrund nicht mehr (nur) auf ihre oft enge Funktion als bergende ,Heimat' beschränkt werden, sondern im Gegenteil als ,offene Regionen“ ausgeweitet werden zu Verdichtungspunkten in ihnen sich überschneidender kultureller Transferbewegungen oft europaweiten Charakters. (JOACHIMSTHALER / RDUCH 2007:1)

\section{Literatur}

[ANONYM] (2002) [=Jürgen Joachimsthaler, Aleksandra Bochenek, Jarosław Bogacki, Ulrike Fügl, Gabriella Jelitto, Grzegorz Jureczko, Maria K. Lasatowicz, Sebastian Mrożek, Daniela Pelka, Michael Rohrwasser, Andrea Rudolph, Izabela Surynt, Magdalena Sutarzewicz, Wiesław Śliwak, Monika Witt, Marek Zybura und Janusz Zydroń] (eds.): Regionalität als Kategorie der Sprach- und Literaturwissenschaft. Hrsg. vom Instytut Filologii Germańskiej der Uniwersytet Opolski. Frankfurt (M.)/Berlin/Bern u. a.

[ANONYM] (2006): III. Interkultureller Kongress. In: Global Visions 12:1f.

ANDERSON, BENEDICT (1993): Imagined Communities. Reflections on the Origin and Spread of Nationalism. London.

ARGONDIZZO, CARMEN (2004): Language Learning through language use. An overview of case studies. Soveria Mannelli.

ASHCROFt, Bill / Griffiths, GARETH / Tiffin, HELEn (eds.) (1989): The Empire Writes Back. Theory and Practice in Post-Colonial Literatures. London/New York.

Bachelard, Gaston (2003): Poetik des Raumes. Aus dem Französischen von Kurt Leohnhard. Frankfurt (M.).

Bachmann-Medick, Doris (2006): Spatial Turn. In: Bachmann-Medick, Doris: Cultural Turns. Neuorientierungen in den Kulturwissenschaften. Reinbek bei Hamburg, 284-328.

BAIER, LOTHAR (1990): Volk ohne Zeit. Essay über das eilige Vaterland. Berlin.

- (2000): Keine Zeit. 18 Versuche über die Beschleunigung. München.

Bнавна, Hомі K. (2000): Die Verortung der Kultur. Mit einem Vorwort von Elisabeth Bronfen. Deutsche Übersetzung von Michael Schiffmann und Jürgen Freudl. Tübingen.

BRAKONIECKI, KAZIMIERZ (1998): Atlantyda pótnocy. Atlantis des Nordens. Zweisprachig. Aus dem Polnischen von Winfried Lipscher. Olsztyn.

BRAKONIECKI, KAZIMIERZ / LIEPSCHER, WinfrIED (eds.) (2001): Meiner Heimat Gesicht. Ostpreußen im Spiegel der Menschen und Landschaft. München.

BRĚZAN, JURIJ (1976): Krabat oder Die Verwandlung der Welt. Berlin.

- (1999): Ohne Paß und Zoll. Aus meinem Schreiberleben. Leipzig.

Castells, Manuel (1996-1998): The Information Age. Economy, Society and Culture. 3 Bde. Oxford. 
Von der einen Nation zur kulturell vielfältigen Region

Chiellino, Carmine (ed.) (2000): Interkulturelle Literatur in Deutschland. Eine Einführung. Stuttgart/Weimar.

Chwin, Stefan (1995): Hanemann. Gdańsk.

- (1997): Tod in Danzig. Aus dem Polnischen von Renate Schmidgall. Reinbek bei Hamburg.

Damir-Geilsdorf, Sabine / Hartmann, Angelika / Hendrich, Béatrice (eds.) (2005): Mental Maps - Raum - Erinnerung. Kulturwissenschaftliche Zugänge zum Verhältnis von Raum und Erinnerung. Münster.

Dangschat, Jens S. (1996): Raum als Dimension sozialer Ungleichheit und Ort als Bühne der Lebensstilisierung? - Zum Raumbezug sozialer Ungleichheit und von Lebensstilen. In: SCHWENK, OTTO G. (ed.): Lebensstil zwischen Sozialstrukturanalyse und Kulturwissenschaft. Opladen, 99-138.

Derdowski, Hieronim (1880): O Panu Czorlińścim co do Pucka po sece jachot. [Wie Herr Czorliński nach Putzig fuhr, um Netze zu kaufen]. Toruń.

DE Stä̈l, Madame (1813). De l'Allemagne. London.

Dzikowska, ElŻBIETa (1997): Terra recognita. Polnische Schriftsteller über deutsche Vergangenheit ihrer schlesischen Heimatorte. In: HONSZA, NORBERT / MECHTENBERG, THEO (eds.): Die Rezeption der deutschsprachigen Gegenwartsliteratur nach der Wende 1989. Wrocław.

Foucault, Michel (2005): Die Heterotopien / Der utopische Körper. Zwei Radiovorträge. Zweisprachige Ausgabe, übersetzt von Michael Bischoff. Mit einem Nachwort von Daniel Defert. Frankfurt (M.).

FRIEDMAN, ThOMAS L. (2008): Die Welt ist flach. Eine kurze Geschichte des 21. Jahrhunderts. Aktualisierte und erweiterte Ausgabe. Aus dem Englischen von Michael Bayer, Hans Freundl, Ekkehard Knörer und Thomas Pfeiffer. Frankfurt (M.).

FunKen, Christiane / Löw, MARTina (eds.) (2003): Raum - Zeit - Medialität. Interdisziplinäre Studien zu neuen Kommunikationstechnologien. Opladen.

GöTZE, LuTZ (2004): Zeitkulturen. Gedanken über die Zeit in den Kulturen. Frankfurt (M.)/Berlin/Bern u. a.

Gould, Peter / Ehite, Rodney ( $\left.{ }^{2} 1986\right)$. Mental maps. London / Sidney.

Graszewicz, Marek / Zybura, MAReK (eds.) (1997): Wroctaw liryczny - lyrisches Breslau. Wrocław.

GRIMBERG, MARTIN (1988): Untersuchungen zum Verlust der Schriftsprachlichkeit. Entstehungsgeschichte, -bedingungen und Einflußfaktoren einer allgemeinen Literalität unter besonderer Berücksichtigung der schriftlich fixierten privaten Kommunikation. Frankfurt (M.) u. a.

GRIMM, JACOB / GRIMM, WiLHELM ( $\left.{ }^{2} 1865\right)$ : Deutsche Sagen. Berlin.

GRZELAK, WoJCIECH (ed.) (2001): Karkonosze liryczne. Lyrisches Riesengebirge. Lyrické Krkonoše. Jelenia Góra. 
Jürgen Joachimsthaler

Hartmann, Regina (2002): Das literarische Selbstbild einer Region - SchwedischPommern im 18. Jahrhundert. In: [ANONYM], 197-214.

[HERDER, JoHANN GOTTFRIED] (ed.) (1773): Von deutscher Art und Kunst. Einige fliegende Blätter. Hamburg [anonym erschienen].

HEYDEBRAND, RENATE VON (1999): Erforschung regionaler Literatur - heute? Überlegungen zu Rechtfertigung und Methodik. In: SCHANDERA, GunTHER / SCHILling, Michael (eds.): Prolegomena zur Kultur- und Literaturgeschichte des Magdeburger Raumes. Magdeburg, 13-31.

Hofmann, Michael (2006): Interkulturelle Literaturwissenschaft. Eine Einführung. Paderborn.

HuElle, PAWEe (1987): Weiser Dawidek. Gdańsk.

- (1995): Weiser Dawidek. Aus dem Polnischen von Renate Schmidgall. Frankfurt (M.).

Huntington, Samuel P. (1996): The Clash of Civilizations. New York.

JOACHIMSTHALER, JÜRGEN (2002): Die Literarisierung einer Region und die Regionalisierung ihrer Literatur. In: [ANONYM], 17-49.

- (2002a): Kulturraumformung durch Sprach- und Literaturpolitik. Mit einführenden Bemerkungen zum „Kulturraum“-Begriff. In: Orbis Linguarum 21:109-129.

- (2005): Text und Raum. In: KulturPoetik 5:243-255.

- (2007): Erfundene Länder. Seh-Barkeit und Bedeutung. In: Moderne. Kulturwissenschaftliches Jahrbuch 2:64-79.

- (о. J.) Die deutschen Litauer. Zur Funktion einer ethnischen Minderheit in der deutschen National-Textur bis 1918. Erscheint in: MAIER, KONRAD (ed.): Sprache und Nation in Nordosteuropa. Wiesbaden.

JOACHIMSTHALER, JÜRGEN / RDUCH, ROBERT (2007): Digitale Bibliothek OS. Eine Vision: http://www.kakanien.ac.at/beitr/materialien/JJoachimsthaler_RRduch1.pdf

KASZYŃSKi, StefAn H. (1987): Galizien - eine literarische Heimat. Poznań.

Ketelsen, Uwe-K. (2006): „Vier Jungens gehen zur See, vier Jungens werden Landwirt irgendwo im Osten. "Die deutsche ,Ostkolonisation" als diskursives Ereignis. In: BALZER, BERND / HAŁUB, MAREK (eds.) (2006): Wroctaw - Berlin. Germanistischer Brückenschlag im deutsch-polnischen Dialog. II. Kongress der Breslauer Germanistik. Bd. 3: Literaturgeschichte 18.-20. Jahrhundert. Hrsg. von Bernd Balzer und Wojciech Kunicki. Wrocław/Dresden, 11-19.

KĘTRZYŃSKI, WoJCIECH (1938): Aus dem Liederbuch eines Germanisierten. Lwów.

KÖSTER, WERNER (2002): Die Rede über den „Raum“. Zur semantischen Karriere eines deutschen Konzepts. Heidelberg.

KoHR, LEOPOLD (1983): Die überentwickelten Nationen. Rückbesinnung auf die Region. Aus dem Englischen übertragen von Walter Schwerdtfeger. München.

KONSTANTINOVIĆ, ZORAN / RINNER, FRIDRUN (2003): Eine Literaturgeschichte Mitteleuropas. Innsbruck/Wien/München/Bozen. 
Koschmal, Walter (1993): Sorbische Literatur in deutscher Sprache. (Original, Übertragung). In: Koschmal, WALTER (ed.): Perspektiven sorbischer Literatur. Köln, 297-310.

Krzyżanowski, Julian / Hernas, CzesŁaw (1985): Literatura polska. Przewodnik encyklopedyczny. [Polnische Literatur. Enzyklopädischer Leitfaden]. Bd. 2. Warszawa.

KÜRSCHNER, JosEPH (ed.) (1896): Das ist des Deutschen Vaterland. Berlin/Eisenach/ Leipzig.

Kunn, Thomas S. (1973): Die Struktur wissenschaftlicher Revolutionen. Aus dem Amerikanischen von Kurt Simon. Frankfurt (M.).

KURPIUn, ROBERT (1909): Der Mutter Blut. Kattowitz.

LÄPPLE, DiETER (1991): Essay über den Raum. In: HÄUSSERMANN, HARTMUT / IPSEN, DetleV / Krämer-Badoni, Thomas (eds.): Stadt und Raum. Soziologische Analysen. Pfaffenweiler, 157-207.

LipPuner, Roland / Lossau, Julia (2004): In der Raumfalle. Eine Kritik des spatial turn in den Sozialwissenschaften. In: MeIN, GEORG / RIEGER-LADICH, MARKus (eds.): Soziale Räume und kulturelle Praktiken. Über den strategischen Gebrauch von Medien. Bielefeld, 47-64

Liskowacki, ARTUR Daniel (2000): Eine kleine. Szczecin.

- (2003): Sonate für S. Aus dem Polnischen von Joanna Manc. München.

LÖW, MARTINA (2001): Raumsoziologie. Frankfurt (M.).

Lotman, JuRI M. (1990): Über die Semiosphäre. In: Zeitschrift für Semiotik 12: 287-305.

Lubos, ARno (1974): Geschichte der Literatur Schlesiens. 3. Bde. München.

MANN, THOMAS (1918): Betrachtungen eines Unpolitischen. Berlin.

Maresch, Rudolf / Werber, Niels (eds.) (2002): Raum - Wissen - Macht. Frankfurt (M.).

OrŁowsKi, Hubert (1996): „Polnische Wirtschaft“. Zum deutschen Polendiskurs der Neuzeit. Wiesbaden.

- (2002): Auf der Suche nach regionaler Identität. BORUSSIA als Programm und Praxis. In: [ANONYM], 153-169.

OSTERHAMmel, JÜRGEN (1998): Die Wiederkehr des Raumes: Geopolitik, Geohistoire und historische Geographie. In: Neue Politische Literatur 43:374-397.

PeKAR, ThOMAs (1998): Imaginäre Geographie. Ernst Jünger und der Orient. Mythos Lektüre - Reise. Dortmund.

Peters, Paul (1990): Heinrich Heine „Dichterjude“. Die Geschichte einer Schmähung. Frankfurt (M.).

PIEPER, RichARD (1987): Region und Regionalismus. Zur Wiederentdeckung einer räumlichen Kategorie in der soziologischen Theorie. In: Geographische Rundschau 39/10:534-539. 
Jürgen Joachimsthaler

PotT, HANS-GEORG (2002): Nationale und regionale Identitäten im Zeitalter der Globalisierung. In: [ANONYM], 113-122.

Rolshoven, Johanna (2003): Von der Kulturraum- zur Raumkulturforschung. Theoretische Herausforderungen an eine Kultur- und Sozialwissenschaft des Alltags. In: Zeitschrift für Volkskunde 99/2:189-213.

RUSHDIE, SALMAN (1990): Ein Reisender über Grenzen im Ich und in der Zeit. Übersetzt von Volker Neuhaus. In: Hermes, Daniela / Neuhaus, Volker (eds.): Günter Grass im Ausland. Texte, Daten, Bilder. Frankfurt (M.), 174-180.

SAID, EDWARD W. (1994): Kultur und Imperialismus. Einbildungskraft und Politik im Zeitalter der Macht. Aus dem Amerikanischen von Hans-Horst Henschen. Frankfurt (M.).

SCHENK, FRITHJof BENJAMIN (2004): The Historical Regions of Europe - Real or Invented? Some Remarks on Historical Comparison and Mental Mapping. In: SCHENK, Frithjof Benjamin (ed.): Beyond the Nation. Writing European History Today. St. Petersburg, 15-24.

SCHLÖGEL, KARL (2003): Im Raume lesen wir die Zeit. Über Zivilisationsgeschichte und Geopolitik. München/Wien.

- (2004): Kartenlesen, Augenarbeit. Über die Fälligkeit des spatial turn in den Geschichts- und Kulturwissenschaften. In: KITTSTEINER, HEINZ DiETER (ed.): Was sind Kulturwissenschaften? 13 Antworten. München, 261-283.

- (2005): Die Wiederkehr des Raums - auch in der Osteuropakunde. In: Osteuropa 3: 5-17.

SCHMitz, Walter (1993): Regionalität und interkultureller Diskurs. Beispiele zur Geschichtlichkeit ihrer Konzepte in der deutschen Kultur. In: THUM, BERND / FINK, GONTHIER-LouIs (eds.): Praxis interkultureller Germanistik. Forschung - Bildung Politik. Beiträge zum II. Internationalen Kongreß der Gesellschaft für Interkulturelle Germanistik. München, 417-438.

Schmitz, Walter / UdolPh, Ludger (eds.) (2001): Tripolis Praga. Die Prager Moderne um 1900. Dresden.

SCHUltZ, HANS-DieTRICH (1997): Räume sind nicht, Räume werden gemacht. Zur Genese „Mitteleuropas“ in der deutschen Geographie. In: Europa Regional 5:2-14.

- (2002): Raumkonstrukte der klassischen deutschsprachigen Geographie des 19./20. Jahrhunderts im Kontext ihrer Zeit. In: Geschichte und Gesellschaft 28:343-374.

SERING, MAX (1893): Die innere Kolonisation im östlichen Deutschland. Leipzig. SŁAWIŃSKI, JANUSZ (1994): Zanik centrali. [Das Verschwinden der Zentrale]. In: Kresy 2:14f.

SOJA, Edward W. (1996): Thirdspace. Journeys to Los Angeles and Other Real-andImagined Places. Cambridge/Oxford.

STASIUK, ANDZEJ (1995): Opowieści galicyjskie. Kraków. 
- (2004): Galizische Geschichten. Aus dem Polnischen von Renate Schmidgall. Frankfurt (M.).

STRUTZ, JOHANN (1992): Komparatistik regional - Venetien, Istrien, Kärnten. In: ZIMA, PETER V.: Komparatistik. Einführung in die Vergleichende Literaturwissenschaft. Unter Mitwirkung von Johann Strutz. Tübingen, 294-331.

STRZELCZYK, FlORENTINE (1999): Un-Heimliche Heimat. Reibungsflächen zwischen Kultur und Nation. München.

StURM, Gabriele (2000): Wege zum Raum. Methodologische Annäherungen an ein Basiskonzept raumbezogener Wissenschaften. Opladen 2000.

TokarczuK, Olga (1999): Dom dzienny, dom nocny. Wałbrzych.

-(2001): Taghaus Nachthaus. Aus dem Polnischen von Esther Kinsky. Stuttgart/ München.

Uhland, Ludwig (1865): Uhlands Schriften zur Geschichte der Dichtung und Sage. Hrsg. von Wilhelm Holland. Bd. 1. Stuttgart.

Wanatowicz, Maria Wanda (1991): Polskość Górnoślasaków w opiniach Polaków innych dzielnic (1922-1939). [Das Polentum der Oberschlesier in den Augen der Polen aus anderen Regionen (1922-1939)]. In: Studia Ślaskie 50:71-85.

WeIgEL, Sigrid (2002): Zum „topographical turn“. Kartographie, Topographie und Raumkonzepte in den Kulturwissenschaften. In: KulturPoetik 2:151-165.

WeIMAR, Klaus (2003): Geschichte der deutschen Literaturwissenschaft. Paderborn.

ZAGAJEWSKI, ADAM (1998): W cudzym pięknie. Kraków.

- (2000): Ich schwebe über Krakau. Erinnerungsbilder. Aus dem Polnischen von Henryk Bereska. München.

ZAWADA, ANDRZEJ (1996): Brestaw. Eseje o miejscach. [Bresław. Essays über Orte]. Wrocław.

Zybura MAReK (1999): Pomniki niemieckiej przeszłości. Dziedzictwo kultury niemieckiej na Ziemiach Zachodnich i Pótnocnych Polski. [Denkmäler deutscher Vergangenheit. Das deutsche Kulturerbe in West- und Nordpolen]. Warszawa.

ŻYTYNIEC, RAFAE (2007): Zwischen Verlust und Wiedergewinn. Ostpreußen als Erinnerungslandschaft der deutschen und polnischen Literatur nach 1945. Osnabrück/ Olsztyn. 